\title{
Effet protecteur des feuilles de Opilia celtidifolia contre l'ulcère induit par l'éthanol chez le rat
}

\author{
Adiaratou TOGOLA $^{1 *}$, Korotimi KARABINTA ${ }^{1}$, Adama DÉNOU ${ }^{2}$, \\ Mahamane HAIDARA ${ }^{2}$, Rokia SANOGO ${ }^{1,2}$ et Drissa DIALLO ${ }^{1,2}$ \\ ${ }^{I}$ Département de Médecine Traditionnelle, Institut National de Recherche en Santé Publique, \\ BP 1746 Bamako, Mali. \\ ${ }^{2}$ Faculté de Pharmacie de l'Université des Sciences des Techniques et Technologies de Bamako, \\ BP 1805 Bamako, Mali. \\ *Auteur Correspondant ; E-mail: togola_adia@hotmail.com; adiatog@yahoo.fr; \\ Tel: (+223) $75344429 / 66790276$
}

\section{RESUME}

Opilia celtidifolia est une plante traditionnellement utilisée contre les plaies et l'ulcère gastro duodénal. Le but de ce travail était d'étudier l'activité antiulcéreuse des extraits aqueux de cette de la plante. Des extraits aqueux, total et épuisé, ont été préparés par décoction. Le décoté total a été testé à 100 et 200 $\mathrm{mg} / \mathrm{kg}$ et le décocté épuisé à $100 \mathrm{mg} / \mathrm{kg}$. Le Sucralfate a été utilisé comme témoin positif à la dose de 1000 $\mathrm{mg} / \mathrm{kg}$ et le groupe non traité a reçu de l'eau distillée à $10 \mathrm{ml} / \mathrm{kg}$. Les extraits ont été administrés par voie intragastrique, une heure après, $0,5 \mathrm{ml}$ d'éthanol à $90 \%$ a été administré aux animaux pour provoquer l'ulcère pendant une heure. Après ce temps, les rats ont été sacrifiés. L'estomac de chaque rat a été ouvert pour observer et compter les ulcères. L'indice d'ulcère a été exprimé et le pourcentage de protection a été calculé. A $100 \mathrm{mg} / \mathrm{kg}$, le décocté épuisé a induit une protection de la muqueuse de $75 \%$, contre $31 \%$ pour le décocté total. Le Sucralfate, utilisé comme témoin a présenté une protection de $87,50 \%$. Ces résultats démontrent que les feuilles de Opilia celtidifolia peuvent intervenir dans le traitement de l'ulcère gastrique. La mise au point d'un phytomédicament contre l'ulcère gastrique à base des feuilles de cette plante est donc envisageable.

(C) 2014 International Formulae Group. All rights reserved.

Mots clés : Opilia celtidifolia, protection, ulcère gastrique.

\section{INTRODUCTION}

L'ulcère gastroduodénal est une affection circonscrite, destructive et progressive qui atteint la muqueuse et la sous muqueuse de l'estomac et du duodénum. L'ulcère, qu'il soit gastrique ou duodénal, entraîne une perte de substance plus ou moins étendue de la paroi digestive qui atteint la couche musculaire. Il guérit en laissant une cicatrice. On le différencie des érosions, abrasions, exulcérations superficielles qui n'atteignent pas la couche musculaire et qui guérissent sans cicatrice (MIB, 2008). L'ulcère gastroduodénal est souvent provoqué par Helicobacter pylori. Le problème de l'infection à $H$. pylori réside sur ses associations à certaines maladies gastroduodénales dont l'ulcère, la gastrite 
chronique, les lymphomes de MALT et le cancer gastrique (Andoulo et al., 2013). La maladie ulcéreuse gastroduodénale touche presque $10 \%$ de la population mondiale, avec une incidence globale de 3 nouveaux cas pour 100.000 habitants. L'ulcère du bulbe est plus fréquent chez l'homme, la parité est respectée pour l'ulcère gastrique. En détail, la maladie ulcéreuse duodénale a une incidence annuelle de 60 à 80.000 nouveaux cas par an. Helicobacter pylori est alors retrouvé dans la muqueuse gastrique 9 fois sur 10. L'incidence annuelle de la maladie ulcéreuse gastrique est quatre fois plus rare (20.000 cas par an), une infection à Helicobacter pyloriy est retrouvée 7 fois sur dix (MIB, 2008).

Plusieurs travaux ont été menés sur cette pathologie au Mali et dans d'autres pays africains. La prévalence de l'ulcère gastroduodénale était de 4,2\% à Goma en République Démocratique du Congo lors d'une étude menée par Buyana en 2013; celle de l'Helicobacter pylori était de $72,5 \% \mathrm{chez}$ les sujets symptomatiques lors d'une étude prospective menée auprès de 203 patients qui ont bénéficié d'une fibroscopie œsogastroduodénale à Yaoundé au Cameroun (Andoulo et al., 2013). Dans une étude faite à l'hôpital régional de Sikasso au Mali, la fréquence des ulcères gastroduodénaux était de 10,88\% (Diarra et al., 2009). Ces études ont toutes été effectuées essentiellement en milieu urbain.

Habituellement, le traitement médical de l'ulcère ne diffère pas, que celui-ci soit gastrique ou duodénal. L'objectif est non seulement de diminuer l'acidité gastrique, ce qui permet aux ulcères de guérir tout en éliminant les douleurs, mais aussi d'éradiquer la bactérie Helicobacter pylori, afin d'éviter que les ulcères ne réapparaissent. Le traitement antiulcéreux idéal devrait atteindre 4 objectifs (MIB 2008): soulager la douleur, accélérer la cicatrisation, prévenir les complications et diminuer la fréquence des récidives.

Le schéma thérapeutique conventionnel repose sur une trithérapie capable d'éradiquer dans $90 \%$ des cas Helicobacter pylori. En effet celle-ci associe un inhibiteur de la pompe à proton et une bithérapie antibiotiqueantiparasitaire. Ces traitements assez efficaces sont dispensés de nos jours dans la plupart des centres de santé. Le traitement des ulcères gastroduodénaux compliqués se fait en deux étapes: médical et chirurgical (MIB, 2008).

Ces traitements conventionnels bien qu'efficaces ne sont pas bien suivis par les patients. Les raisons entre autres étant leur coût élevé, ce qui fait qu'ils ne sont pas accessibles à une très forte majorité de la population vivant en milieux rural. Ces patients utilisent les ressources de la médecine traditionnelle comme alternative de traitement.

La médecine traditionnelle reste une option à grande potentialité thérapeutique des ulcères de façon générale. De nombreuses plantes et recettes traditionnelles possèdent une propriété curative sur les ulcères. Des études expérimentales et cliniques menées par le Département de Médecine Traditionnelle au Mali ont permis la mise au point de deux phytomédicaments contre les ulcères gastrique et duodénal, appelés médicaments traditionnels améliorés (MTA). Le Gastrosédal à base de racines de Vernonia kotschyana (Asteraceae) (Germano' et al., 1996) et le Calmogastryl à base de l'écorce de tronc de Pteleopsis suberosa (Combrétacées) (Mariko 1989). D'autres plantes ont aussi fait l'objet d'étude contre l'ulcère mais qui ne sont pas encore élaborées en phytomédicament notamment, Cola cordifolia (Malvacées), une plante dont les feuilles et l'écorce sont utilisés traditionnellement contre les douleurs, les plaies et l'ulcère gastroduodénal. Les extraits de cette plante ont démontré un pouvoir significatif d'inhibition de la formation de l'ulcère chez les rats (Austarheim et al., 2012).

Opilia celtidifolia, qui est l'objet de la présente étude, est aussi utilisé en médecine traditionnelle comme cicatrisant des plaies et ulcères (Togola et al., 2005). Les polysaccharides isolés des feuilles de la plante 
ont démontré une puissante propriété d'activation du système immunitaire (Togola et al., 2008). Récemment, les propriétés cicatrisantes d'une pommade formulée avec les polysaccharides de la plante ont été démontrées sur des plaies expérimentales chez le lapin (Togola et al., 2014). L'objectif $\mathrm{du}$ présent travail était donc de tester l'activité protectrice des extraits aqueux total et épuisé des feuilles de Opilia celtidifolia contre l'ulcère induit par l'éthanol chez les rats.

\section{MATERIEL ET METHODES Matériel végétal}

L'étude a été réalisée sur les feuilles de Opilia celtidifolia récoltées à Blendio dans la région de Sikasso. Celles-ci ont été identifiées par un botaniste par comparaison avec un spécimen enregistré sous le numéro 0904DMT. Le matériel a été séché à l'ombre et pulvérisé en poudre fine avec un moulin électrique.

\section{Matériel animal}

Les animaux utilisés étaient des rats blancs Wistar, mâles et femelles, de poids compris entre 90 et 205 g. Au total, cinq lots de cinq rats ont été constitués à raison d'un lot pour chaque dose de substance à tester.

\section{Caractères microscopiques de la poudre de feuilles de $\boldsymbol{O}$. celtidifolia}

L'observation de la poudre a été effectuée à l'objectif 10 avec un microscope ordinaire. Le réactif de Gaz et du Chatelier a été utilisé pour l'immersion.

\section{Dosages de l'eau et des cendres}

Les teneurs en eau et cendres de la poudre de feuilles de Opilia celtidifolia ont été déterminées par les méthodes décrites par la pharmacopée Africaine (WHO, 2011). Le dosage de l'eau consistait à déterminer l'humidité résiduelle présente dans la poudre de la plante, par la mesure de la perte en eau par dessiccation à l'étuve à $103 \pm 2{ }^{\circ} \mathrm{C}$ pendant $24 \mathrm{~h}$, ou par entrainement par un azéotrope.
Le dosage des cendres se fait après calcination totale de la poudre de feuilles à $600{ }^{\circ} \mathrm{C}$ dans un four.

\section{Déterminationdes constituants chimiques}

La caractérisation des différents groupes chimiques, présents dans la matière végétale, a été réalisée par des réactions colorées en tube comme précédemment décrites (Somboro et al., 2011). Les alcaloïdes ont été extraits en milieu acide et précipités par le réactif de Dragendorff (solution aqueuse d'iodo-bismuthate de potassium). Les flavonoïdes ont été révélés par la réaction de la cyanidine; l'ajout d'alcool chlorhydrique (alcool à $95 \%$ et acide chlorhydrique concentré à parties égales en volumes), d'alcool iso-amylique et quelques copeaux de magnésium, provoque une coloration rougeâtre en présence de flavonoïdes. La présence des saponines a été déterminée par la mesure de l'indice de mousse. Les tanins ont été caractérisés par coloration avec une solution aqueuse diluée de chlorure ferrique, qui donne une coloration verdâtre ou bleu noirâtre en présence de ces composés. La teneur des groupes chimiques identifiés étaient évaluée selon l'intensité de la coloration observée lors de la réaction.

\section{Préparation des extraits \\ Décocté total}

Une décoction a été faite avec $270 \mathrm{~g}$ de poudre sèche et $2700 \mathrm{ml}$ d'eau distillée, dans un ballon de décoction pendant $2 \mathrm{~h}$. Après ce temps, le décocté a été filtré, concentré au Rotavapor et lyophilisé. L'extrait sec a ensuite été pesé pour calculer le rendement et conservé dans un dessiccateur.

\section{Décocté épuisé}

Une quantité (460 g) de matière végétale a été successivement épuisée avec $2140 \mathrm{ml}$ de dichlorométhane et $1840 \mathrm{ml}$ d'éthanol utilisant le Soxhlet. La poudre épuisée a été séchée pendant plusieurs heures à la température du laboratoire et utilisée pour une décoction (décoction épuisée) à raison de $100 \mathrm{~g}$ pour 21 d'eau distillée pendant $2 \mathrm{~h}$. Le 
décocté a été ensuite filtré, concentré et lyophilisé. Les lyophilisats obtenus ont été pesés pour calculer le rendement et conservés dans un dessiccateur.

\section{Détermination de l'activité antiulcéreuse gastrique}

Le test consistait à vérifier l'action protectrice des extraits contre l'ulcère provoqué chez les animaux par administration d'un agent ulcérogène. Les Substances à tester étaient le décoté total aux doses de 100 et $200 \mathrm{mg} / \mathrm{kg}$, le décocté épuisé à la dose de 100 mg/kg, le Sucralfate, utilisé comme contrôle positif, à la dose de 1000 $\mathrm{mg} / \mathrm{kg}$ et l'eau distillée, comme contrôle négatif, à la dose de $10 \mathrm{ml} / \mathrm{kg}$

Les rats ont été mis à jeun pendant 24 heures avant l'expérimentation mais avaient accès libre à l'eau. Au début du test, les rats ont reçu par voie intra-gastrique les différents extraits aqueux préalablement dissouts dans de l'eau distillée. Une heure après les traitements, chaque rat a reçu $0,5 \mathrm{ml}$ d'éthanol à 90\% selon la méthode décrite par Germano' et al. (1996). Une heure après l'administration de l'agent ulcérogène, les rats ont été sacrifiés. L'estomac de chaque rat a été prélevé, ouvert selon la grande courbure à l'aide d'un ciseau, lavé avec une solution physiologique, puis fixé dans une solution de formol pendant 5 minutes. Après ce temps, chaque estomac a été bien étalé sur une tablette pour mieux observer les ulcères formés à l'œil nu et à l'aide d'une loupe.

La longueur de chaque ulcère a été mesurée à l'aide d'une règle graduée en $\mathrm{mm}$ Le nombre et la gravité des ulcères ont été déterminés selon une échelle arbitraire de 0 à 6:0= pas de lésion; $1=1$ à 3 petites lésions $\leq$ $10 \mathrm{~mm} ; 2=1$ à 3 larges lésions $\geq 10 \mathrm{~mm} ; 3=$ 1 à 3 lésions épaisses; 4= plus de 3 petites lésions; 5 = plus de 3 larges lésions; $6=$ plus de 3 lésions épaisses (Germano' et al., 1996).

L'indice d'ulcère (IU) de chaque estomac a été exprimé comme la somme des points et le pourcentage de protection (PP) a été calculé par rapport au groupe témoin selon la formule suivante :

$$
\mathrm{PP}=\frac{\mathrm{IU} \text { témoin }-\mathrm{IU} \text { traité }}{\mathrm{IU} \text { témoin }} \times 100
$$

\section{Analyse statistique}

Les résultats ont été analysés par le test $\mathrm{t}$ de Student. Une valeur de $\mathrm{P}<0,05$ était considérée comme significative.

\section{RESULTATS \\ Caractères microscopiques de la poudre de feuilles de Opilia celtidifolia}

L'analyse au microscope de la poudre de feuilles a permis d'observer de très nombreuses fibres, de cristaux d'oxalates de calcium, de grains d'amidons et de fragments d'épiderme de même qu'une présence modérée de cellules périspermiques et duparenchyme cortical.

\section{Teneur en eau, en cendre et en substances extractibles par l'eau}

La teneur en eau par la méthode pondérale et gravimétrique, les teneurs en cendres (totales, chlorhydriques et sulfuriques) et la quantité de substances extractibles par l'eau des feuilles de Opilia celtidifolia sont présentées dans le Tableau 1. La teneur en eau était faible selon les deux méthodes utilisées, une moyenne de teneur en eau de $5 \%$ a été obtenue.

\section{Groupes chimiques identifiés dans la poudre de feuilles de Opilia celtidifolia \\ Les constituants majoritaires} caractérisés par les réactions en tubes ont été les saponosides, les oses et les coumarines (Tableau 2). Les groupes chimiques comme les tanins, flavonoïdes, alcaloïdes, caroténoïdes, anthracénosides, composés réducteurs, hétérosides cardiotoniques, hétérosides cyanogénétiques, anthocyanes et de leuco-anthocyanes noont pas été détectés selon les techniques utilisées.

\section{Rendement des extractions et caractéristiques des extraits \\ Le rendement, la couleur et l'aspect de l'extrait aqueux total et épuisé sont présentés dans le Tableau 3. L'extrait total avait une}


couleur marron-verdâtre un peu terne liée à la présence de composés lipophiles; ces derniers ont été épuisés avant la préparation de l'extrait épuisé, d'où l'absence de la nuance verdâtre et la présence d'une teinte de brillance pour cet extrait.

\section{Activité antiulcéreuse}

L'étude a permis d'évaluer le degré de protection de la muqueuse gastroduodénale par les extraits aqueux contre les ulcérations causées par l'éthanol à 90\%. Les indices d'ulcères et les pourcentages de protection sont présentés dans le Tableau 4. Le décocté épuisé, avec $75 \%$ de protection de la muqueuse gastrique, a été plus actif que le décocté total, avec $31 \%$ de protection, à la même dose $(100 \mathrm{mg} / \mathrm{kg})$. Le Sucralfate utilisé comme témoin et qui était dix fois plus dosé $(1000 \mathrm{mg} / \mathrm{kg})$ a induit une protection de la muqueuse gastrique de $87 \%$.

Tableau 1:Teneur en eau, en cendre, et en substances extractibles par l'eau des feuilles de Opilia celtidifolia.

\begin{tabular}{lc}
\hline Dosage & Résultats (\%) \\
\hline Substances extractibles par l'eau & 14 \\
\hline Teneur en eau: & \\
Méthode gravimétrique & 4,48 \\
Méthode azéotropique & 6 \\
\hline Cendres: & \\
Totales & 16,39 \\
Chlorhydriques & 4,61 \\
Sulfuriques & 23,67 \\
\hline
\end{tabular}

Tableau 2: Groupes chimiques identifiés dans la poudre de feuilles de Opilia celtidifolia.

\begin{tabular}{ll}
\hline Groupes chimiques & Résultats \\
\hline Coumarines & ++ \\
Oses et Holosides & ++++ \\
Polyuronides (mucilages) & ++++ \\
Stérols et Triterpènes & ++ \\
\hline Saponosides & \\
Mousse & ++++ \\
Indice de mousse & 1000 \\
\hline++++ Réaction très positive & \\
++ Réaction moyennement positive &
\end{tabular}

Tableau 3: Caractéristiques des décoctés aqueux, total et épuisé, des feuilles de Opilia celtidifolia.

\begin{tabular}{lccccc}
\hline Extraits & $\begin{array}{c}\text { MPE } \\
(\mathbf{g})\end{array}$ & $\begin{array}{c}\text { Masse } \\
\text { d'extrait }(\mathbf{g})\end{array}$ & $\begin{array}{c}\text { Rendements } \\
\mathbf{( \% )}\end{array}$ & Couleur & Aspect \\
\hline Décocté total & 270 & 48,94 & 18,12 & Marron verdâtre & Terne avec quelques cristaux \\
Décocté épuisé & 300 & 43,16 & 14,39 & Marron & Brillant avec cristaux \\
\hline
\end{tabular}


Tableau 4: Activité anti ulcère gastrique du décocté total (DT) et du décocté épuisé (DE) de feuilles de Opilia celtidifolia contre l'ulcère provoqué par l'éthanol 90\% pendant 60 minutes chez les rats.

\begin{tabular}{lccc}
\hline Produits & Doses/kg & Indice d'Ulcère (IU \pm DS) & Protection(\%) \\
\hline Eau distillée & $10 \mathrm{ml}$ & $3,20 \pm 1,09$ & --- \\
Décocté total & $100 \mathrm{mg}$ & $2,20 \pm 1,64 \mathrm{NS}$ & 31,25 \\
Décocté total & $200 \mathrm{mg}$ & $0,60 \pm 0,37 * *$ & 81,25 \\
Décocté épuisé & $100 \mathrm{mg}$ & $0,80 \pm 0,27 * *$ & 75,00 \\
Sucralfate (Témoin) & $1000 \mathrm{mg}$ & $0,40 \pm 0,54 * *$ & 87,50 \\
\hline Les résultats sont exprimés en moyenne de l'indice d'ulcère plus ou moins la déviation standard (IU) \pm (DS), La \\
significativité est exprimée par: NS = non significatif; **très significatif avec P<0,01 (test t de Student)
\end{tabular}

\section{DISCUSSION}

Opilia celtidifolia est une plante bien connue par les tradipraticiens de santé au Mali, pour le traitement de plusieurs maladies comme le paludisme, les dermatoses, les douleurs abdominales, le manque d'appétit, les plaies, les vers intestinaux (Togola et al., 2005).

Le choix de cette plante, pour une investigation des propriétés antiulcéreuse des feuilles, a été motivé par plusieurs constats: ses feuilles possèdent des propriétés cicatrisantes des plaies et l'ulcère en Médecine Traditionnelle (Togola et al., 2005; Diallo et al., 2002); elles contiennent des polysaccharides ayant une propriété d'activation de plusieurs composantes du système immunitaire notamment le complément, les macrophages, les lymphocytes B et T (Togola et al., 2008); et le décocté épuisé des feuilles et ses fractions ont une propriété cicatrisante confirmée sur les plaies externes (Togola et al., 2014). De nombreux travaux ont démontré que les plantes utilisées traditionnellement comme cicatrisant possèdent des propriétés d'activation du système immunitaire; cette activation serait due à la présence des polysaccharides qui seraient responsables de la guérison des plaies externes et de l'ulcère gastroduodénal (Nergard, 2004).

La détermination des caractéristiques microscopiques des feuilles de la plante ont permis une meilleure identification de la matière végétale et la détection d'éventuelles falsifications. Une faible teneur en eau (inférieure à $10 \%$, normes établies par la pharmacopée internationale), est recommandée pour la bonne conservation des végétaux; la teneur en eau de la poudre était dans les normes. Ce taux empêche les réactions d'oxydation, de fermentation et la formation de moisissures.

Les cendres totales (16,39\%) renseignent sur la charge en éléments minéraux, les cendres sulfuriques $(23,67 \%)$ résultent de la conversion de sels organiques en sulfates et les cendres chlorhydriques $(4,61 \%)$ renseignent sur les matières siliceuses.

La teneur de substances extractibles par l'eau (environ 14\%) exprime la quantité de substances solubles dans l'eau. L'importante de cette teneur est grande dans le cadre de la présente étude car les substances d'intérêt sont les polysaccharides hydrosolubles.

L'étude phytochimique a permis de déterminer la présence modérée de coumarines, les stérols et triterpènes et la présence en proportion très élevée de saponosides, de mucilages, d'oses et holosides. Ces différents résultats sont similaires à ceux de Sangaré (2004). Selon la littérature, ces composés identifiés peuvent conférer plusieurs propriétés biologiques à la plante. Les composés terpéniques (stérols et triterpènes) confèrent une propriété antipyrétique, souvent analgésique et 
antiinflammatoire (Bruneton, 1999). Les saponosides sont utilisés par plusieurs végétaux dans leur système de défense antimicrobienne. Le grand intérêt de ces composés dans l'activité antiulcéreuse gastrique est l'élimination de Helicobacter pylori. Certains saponosides également confèrent à la plante des propriétés antiinflammatoire et antalgique (Bruneton 1999), ce qui est également bénéfique dans le cadre de l'ulcère gastrique. Les mucilages sont très recherchés pour leurs propriétés cicatrisantes et gastro-protectrices (Yansambou 2002). Opilia celtidifolia n’a pas fait l'objet de plusieurs études d'activités biologiques selon la littérature, mais il existe une concordance entre les activités biologiques des groupes chimiques qui ont été identifiés dans nos résultats avec les utilisations traditionnelles recueillies lors des enquêtes ethnobotaniques antérieures (Togola et al., 2005).

Le décocté total et le décocté épuisé de la plante, administrés par voie intra-gastrique à des doses différentes, ont protégé la muqueuse gastrique contre les ulcérations provoquées par l'éthanol à $90 \%$ chez les rats. Dans nos conditions expérimentales, le décocté épuisé a présenté une meilleure protection de la muqueuse gastrique que le décocté total à la même dose. Ceci pourrait être dû à une abondance de polysaccharides dans l'extrait épuisé comme signalé dans d'autres études (Austarheim et al., 2012; Togola et al., 2008). Les polysaccharides peuvent avoir une propriété de protection de la muqueuse gastrique (pansement gastrique) de par leur viscosité et aussi une propriété de guérison de la maladie proprement dite. Le Sucralfate utilisé dans ce test comme témoin est également un polysaccharide. Les polysaccharides d'autres plantes comme Cola cordifolia et Vernonia kotschyana, ont aussi inhibé de manière significative la formation de lésions gastriques chez les rats (Austarheim et al., 2012). Tout comme Opilia celtidifolia, ces deux plantes sont utilisées dans le traitement traditionnel des insuffisances digestives, des affections gastriques et de l'ulcère gastroduodénal au Mali. Les résultats obtenus avec Opilia celtidifolia apportent donc une justification à l'utilisation traditionnelle des extraits aqueux de la plante dans le traitement de l'ulcère gastrique.

\section{Conclusion}

Les polysaccharides, probablement en synergie avec les autres constituants des feuilles de Opilia celtidifolia, peuvent intervenir dans le traitement de l'ulcère gastrique. La mise au point d'un nouveau phytomédicament contre l'ulcère gastrique à base des feuilles de Opilia celtidifolia est donc envisageable pour une meilleure prise en charge de cette affection au Mali.

\section{REFERENCES}

Andoulo FA, Noah DN, Tagni-Sartre M, Ndam ECN, Blackett KN. 2013. Epidémiologie de l'infection à Helicobacter Pylori à Yaoundé: de la particularité à l'énigme Africaine. Pan Afr. Med. J., 16(115) doi:10.11604/ pamj.2013.16.115.3007

Austarheim I, Haidara M, Sanogo R, Togola A, Mhaledadi M, Vestrheim AC, Inngierdingen KT, Michaelsen TE, Diallo D, Paulsen BS. 2012. Antiulcer polysaccharides from Cola cordifolia bark and leaves. J. Ethnopharm.,143(1): 221-227.

Buyana A. 2013. Ulcère Gastrique dans la ville de Goma. Thèse de Médecine, Université de Goma, Répblique Démocratique du Congo, p. 38.

Bruneton J. 1999. Pharmacognosie Phytochimie Plantes Médicinales ( 3 ème edn). Edition Technique et Documentation, Lavoisier: Paris.

Diallo D, Sogn C, Samaké FB, Paulsen BS, Michaelsen TE, Keita A. 2002. Wound healing plants in Mali, the Bamako region: an ethnobotanical survey and complement fixation of water extracts from selected plants. Pharm. Biol., 40: 117-128. 
Diarra M, Konate A, Traore C, Soukho A, Kamate B, Diallo AB, Maiga MY. 2009. Les ulcères gastroduodénaux en milieu rural. Mali Méd.XXIV(2): 1-3.

Germano' MP, De Pasquale R, Iauk L, Galati EM, Keita A, Sanogo R. 1996. Antiulcer activity of Vernoniakotschyana Sch. Bip. Phytomed., 2(3): 229-233.

Mariko MD. 1989. Etude de l'activité de " TERENIFOU" écorce de tronc de Pteleopsissuberosa Engl. et Diels (Combretaceae) dans le traitement des ulcères gastroduodénaux, Thèse de Médecine, Université de Bamako, Mali, p. 87.

MIB N²90 2008. Ulcère gastrique et duodénal, gastrite. Faculté de Médecine Monpellier-Nîmes, LIPCOM-RM: p14. Disponible à : http://www.med.univmontp1.fr/enseignement/cycle_2/MIB/Re ssources_locales/App-Digest/MIB_290_ ulcere_gastrique_duodenal_gastrite_word .pdf

Nergård CS. 2005. Immunomodulating pectic polymers, Thesis for the degree of Doctor Scientiarum, University of Oslo, Norway, p. 80 .

Sangaré D. 2004. Etude de la prise en charge $\mathrm{du}$ paludisme par les thérapeutes traditionnels dans les aires de santé de Kendie (Bandiagara) et de Finkolo (Sikasso). Thèse de Pharmacie, Université de Bamako, Mali, p. 131.

Somboro AA, Patel K, Diallo D, Sidibe L, Chalchat JC, Figueredo G, Ducki S, Troin Y, Chalard P. 2011. An ethnobotanical and phytochemical study of the African medicinal plant Guiera senegalensis J. F. Gmel. J. Med. Plant Res., 5(9): 16391651.

Togola A, Karabinta K,Dénou A, Haidara M, Sanogo R, Diallo D. 2014. Polysaccharides cicatrisants isolés des feuilles de Opilia celtidifolia (Guill. et Perr.) Endl. ex Walp. (Opiliacées) in press Mali Méd.

Togola A, Inngjerdingen M, Diallo D, Barsett H, Rolstad B, Michaelsen TE, Paulsen BS. 2008. Polysaccharides with complement fixing and macrophage stimulation activity from Opilia celtidifolia, isolation and partial characterization. J. Ethnopharm., 115: 423-431.

Togola A, Diallo D, Dembele S, Barsett H, Paulsen BS. 2005. Ethnopharmacological survey of different uses of seven medicinal plants from Mali, (West Africa) in the regions Doila, Kolokani and Siby. J. Ethnobiol. Ethnomed., 1: 715.

World Health Organization 2011. Quality Control Methods for Herbal Materials ( $2^{\text {nd }}$ edn). WHO Library CataloguingPublication Data: Malta.

Yansambou H. 2002. Etude des constituants des feuilles de Zizyphusmauritiana Lam. (Rhamnacées) utilisées traditionnellement dans le traitement du diabète au Mali. Thèse de Pharmacie, Université de Bamako, Mali, p. 89. 